\title{
1 Scaling between stomatal size and density in forest plants
}

2

3 Congcong Liu ${ }^{1,2 \#}$, Christopher D. Muir ${ }^{3 \#}$, Ying $\mathrm{Li}^{1}, \mathrm{Li} \mathrm{Xu}^{1}, \mathrm{Mingxu} \mathrm{Li}{ }^{1}$, Jiahui Zhang ${ }^{1,2}$, Hugo

4 Jan de Boer ${ }^{4}$, Lawren Sack ${ }^{5}$, Xingguo Han $^{6}$, Guirui Yu ${ }^{1,2}$, Nianpeng $\mathrm{He}^{1,2,7^{*}}$

5

$6{ }^{1}$ Key Laboratory of Ecosystem Network Observation and Modeling, Institute of Geographic

7 Sciences and Natural Resources Research, Chinese Academy of Sciences, Beijing 100101,

8 China

$9{ }^{2}$ University of Chinese Academy of Sciences, Beijing 100049, China

$10{ }^{3}$ School of Life Sciences, University of Hawai’i at Mānoa, Honolulu, 96822, USA

$11{ }^{4}$ Copernicus Institute of Sustainable Development' Department of Environmental Sciences,

12 Utrecht University, the Netherlands, 80125, Utrecht.

$13{ }^{5}$ Department of Ecology and Evolutionary Biology, University of California, Los Angeles,

1490025 , USA

$15{ }^{6}$ State Key Laboratory of Vegetation and Environmental Change, Institute of Botany, Chinese

16 Academy of Sciences, Beijing 100093, China

$17{ }^{7}$ Institute of Grassland Science, Northeast Normal University, and Key Laboratory of Vegetation

18 Ecology, Ministry of Education, Changchun 130024, China

19

20 \# These authors contributed equally to this work.

$21 *$ Corresponding author Nianpeng He (henp@igsnrr.ac.cn).

22 Tel.: +86-10-64889263

23 Fax: +86-10-64889399 


\section{Abstract}

25 The size and density of stomatal pores limit the maximum rate of leaf carbon gain and

26 water loss $\left(g_{\max }\right)$ in land plants. The limits of $g_{\max }$ due to anatomy, and its constraint by the

27 negative correlation of stomatal size and density at broad phylogenetic scales, has been

28 unclear and controversial. The prevailing hypothesis posits that adaptation to higher $g_{\text {max }}$ is

29 typically constrained by geometry and/or an economic need to reduce the allocation of

30 epidermal area to stomata (stomatal-area minimization), and this would require the

31 evolution of greater numbers of smaller stomata. Another view, supported by the data, is

32 that across plant diversity, epidermal area allocated to guard cells versus other cells can be

33 optimized without major trade-offs, and higher $g_{\max }$ would typically be achieved with a

34 higher allocation of epidermal area to stomata (stomatal-area increase). We tested these

35 hypotheses by comparing their predictions for the structure of the covariance of stomatal

36 size and density across species, applying macroevolutionary models and phylogenetic

37 regression to data for 2408 species of angiosperms, gymnosperms, and ferns from forests

38 worldwide. The observed stomatal size-density scaling and covariance supported the

39 stomatal-area increase hypothesis for high $g_{\text {max }}$ A higher $g_{\max }$ involves construction costs

40 and maintenance costs that should be considered in models assessing optimal stomatal

41 conductance for predictions of water use, photosynthesis, and water-use efficiency as

42 influences on crop productivity or in Earth System models. 
Stomatal pores are critical determinants of the function of plants and the composition of

45 the atmosphere (1). The stomatal conductance to diffusion of water vapor and $\mathrm{CO}_{2}\left(g_{\mathrm{s}}\right)$

46 influences a broad spectrum of ecological processes at leaf, community, and ecosystem scales,

47 including photosynthesis, net primary production, and water use efficiency $(2,3)$. Theoretically,

48 stomata can regulate $g_{\mathrm{s}}$ through evolutionary or plastic shifts in stomatal size or numbers (4) or

49 through short-term stomatal aperture changes (5). The $g_{\mathrm{s}}$, and its typical operational value $\left(g_{\text {op }}\right)$,

50 can thus vary from near zero with stomata fully closed and $g_{\max }$ with stomata fully open. The

$51 g_{\max }$ is a fundamental anatomical constraint, and across species measured under controlled

52 conditions, $g_{\text {op }}$ and $g_{\max }$ are correlated $(6,7)$. Because of their importance in controlling leaf

53 water and $\mathrm{CO}_{2}$ fluxes, stomatal anatomy can provide critical information in global vegetation and

54 crop models (8-11) toward the current grand challenge of understanding how crops and forest

55 trees are optimized for carbon gain versus water use. Yet, there has been substantial debate about

56 the anatomical underpinnings of the evolution of higher $g_{\max }$, and its associated costs.

57 The $g_{\max }$ is a mathematic function of underlying anatomical traits stomatal density $\left(D_{\mathrm{s}}\right.$,

58 number of pores per unit epidermal area) and size $\left(A_{\mathrm{s}}\right.$, area of guard cells surrounding each pore).

59 Indeed, these traits are widely used to study the adaptation and competition of plants because

60 they are reliable indicators of $g_{\max }(12-18)$. Further an inverse relationship between $A_{\mathrm{s}}$ and $D_{\mathrm{s}}$

61 across diverse plant species has been recognized since 1865 (19). A prevalent view in the

62 literature established by Franks and Beerling (20) is that the negative $A_{\mathrm{s}}$ and $D_{\mathrm{s}}$ relationship and

63 the cost of stomatal area place a strong constraint on the evolution of $g_{\max }$. According to an early

64 version of the "stomatal area minimization hypothesis" a packing limit geometry constrains $g_{\max }$,

65 because the total fraction of epidermal area allocated to stomata $\left(f_{s}\right)$ cannot exceed unity:

$$
\boldsymbol{f}_{S}=\boldsymbol{D}_{\mathrm{S}} \boldsymbol{A}_{\mathrm{S}} .
$$


This, in turn generates the negative $A_{\mathrm{s}}$ and $D_{\mathrm{s}}$ relationship such that the evolution of larger

67 numbers of stomata would necessitate reduction in their size. Thus, higher $g_{\max }$ can only be

68 achieved by the evolution of larger numbers of smaller stomata. This early packing geometry

69 argument was rendered moot given observations that for functional leaves, maximum $f_{s}$ is

70 usually far lower than unity (33.6\% in our data; solid line in Fig. 1a). First, because stomata need

71 to be spaced out by epidermal cells to open and close properly (21), and second, because the

72 development of higher $D_{\mathrm{s}}$ can occur through the increased differentiation of epidermal cells into

73 stomata (i.e., achieving higher stomatal differentiation rate, or stomatal index $(22,23)$, such that

74 stomatal numbers can be independent of sizes. Yet the stomatal area minimization hypotheses for

75 the evolution of higher $g_{\max }$ and its association with the negative $A_{\mathrm{s}}$ and $D_{\mathrm{s}}$ relationship was

76 reached by a different argument: that to minimize stomatal construction and maintenance costs

77 (24), plants evolving higher $g_{\max }$ must do so with a reduced $f_{s}$, and this maximization of $g_{\max }$

78 relative to $f_{s}$ would in turn generate the negative $A_{\mathrm{s}}$ and $D_{\mathrm{s}}$ relationship (20).

79 To see why, note that a leaf's $g_{\max }$ is determined by stomatal anatomy:

$$
g_{\max }=b m D_{\mathrm{S}} A_{\mathrm{S}}^{0.5}
$$

80 where $b$ and $m$ are biophysical and morphological constants, respectively (22) (see Methods for

81 equations to calculate these constants). By Eq. 2, a higher $g_{\max }$ can be achieved with a smaller

82 total stomatal area by increasing stomatal number and reducing stomatal size, because smaller

83 stomata also have a shorter channel for diffusion. For example, consider two leaves with

84 stomatal densities 250 and 200 pores $\mathrm{mm}^{-2}$ and stomatal areas $150 \mu \mathrm{m}^{2}$ and $187.5 \mu \mathrm{m}^{2}$. They

85 have identical $f_{\mathrm{S}}$, but $g_{\max }$ at $25{ }^{\circ} \mathrm{C}$ is $11 \%$ greater for the leaf with smaller stomata (1.32 versus

$861.47 \mathrm{~mol} \mathrm{~m}^{-2} \mathrm{~s}^{-1}$ ). Thus, selection for higher $g_{\max }$ would result in more numerous, smaller 
87 stomata, to minimize epidermal allocation to stomata and the evolution of higher $g_{\max }$ is strongly

88 associated with the negative $A_{\mathrm{s}}$ and $D_{\mathrm{s}}$ relationship.

The ‘stomatal-area minimization' hypothesis is controversial, however, as it is at odds

90 with data in the literature that instead support an opposite, 'stomatal-area increase' hypothesis,

91 i.e., that $g_{\max }$ should increase with $f_{\mathrm{S}}$ during evolution. Conversely, selection decreased $g_{\max }$

92 would be associated with decreased $f_{\mathrm{S}}$. The positive covariance of $g_{\max }$ with $f_{\mathrm{S}}$ has been shown in

93 many studies and has been utilized in many papers in the literature that have indeed used $f_{\mathrm{S}}$ as a

94 proxy for $g_{\max }(25,26)$. According to the "stomatal-area increase" hypothesis, selection for

95 higher $g_{\max }$ is much stronger than that to minimize cost, leading to greater surface allocation,

96 even if this incurs a cost. Under this scenario, the negative $A_{\mathrm{s}}$ and $D_{\mathrm{s}}$ relationship would not act

97 directly as a constraint on $g_{\max }$. Yet, selection for higher $g_{\max }$ would generate a distinctive

98 covariation between these constituent anatomical traits. First, no relationship of $A_{\mathrm{s}}$ and $D_{\mathrm{s}}$ is

99 absolutely required, which is consistent with data in the literature for species sets for which no

100 relationship is found (27); as less than $50 \%$ of the leaf surface is typically taken up by stomata

101 and many qualitatively different relationships between stomatal size and density across species

102 are geometrically possible, including negative, zero, and positive covariances (ellipses in Fig.

103 1a). Yet, on average, a specific covariation would be expected if many combinations of $A_{\mathrm{s}}$ and $D_{\mathrm{s}}$

104 have similar fitness through their effect on either $g_{\max }$ or $f_{\mathrm{S}}$, as we derive below.

105

It is critical to distinguish between these hypotheses for the evolution of $g_{\max }$ and the

106 potential for $f_{\mathrm{S}}$ to constrain the observed stomatal size-density relationship. Implications of

107 stomatal-area minimization are that $g_{\max }$ is ultimately constrained by the costs of high $f_{\mathrm{S}}$, that

108 such costs are minimized, and that evolving higher $f_{\mathrm{S}}$ would be slowed by costs associated with

109 allocating too much epidermal area to stomata ${ }^{27-32}$. By contrast, the stomatal-area increase 
110 hypothesis implies that selection on stomatal size and density primarily optimizes $g_{\max }$, which

111 varies across environments, and greater $g_{\max }$ incurs stomatal construction costs and opportunity

112 costs of epidermal space. Testing these hypotheses will further reveal how the evolution of high

$113 g_{\max }$ relates to the general inverse stomatal size-density relationship.

114 Indeed, these hypotheses can be tested against data for diverse species by considering in

115 detail the covariation among $D_{\mathrm{S}}$ and $A_{\mathrm{S}}$, for which they make different predictions. Under both

116 hypotheses, $D_{\mathrm{S}}$ and $A_{\mathrm{S}}$ are constituents of composite traits, $f_{\mathrm{S}}$ or $g_{\max }$ (Eq. 1-2; Fig. 1b). We

117 investigated how stomatal size-density scaling would differ between the hypotheses using

118 models of macroevolutionary landscapes (28-31). We used the Ornstein-Uhlenbeck (OU) model

119 originally derived from quantitative genetics for intraspecific (population) trait microevolution

120 by Lande (32), and developed by Hansen (29)and others (28) for macroevolutionary interspecific

121 trait variation. In the macroevolutionary OU model, interspecific trait variation expands through

122 time until it reaches a stationary distribution around a long-term average(29). Within each

123 species, microevolutionary forces (selection, genetic drift, mutation, and migration) and the

124 environment drive genetic and plastic trait variation, respectively, and species' trait means

125 should be near their current adaptive optimum. The across-species distribution that becomes

126 stationary in the OU model is thus dependent on these independent shifts in species' optimum

127 trait values. At stationarity, an OU process leads to stable trait mean and variance, setting the

128 overall phenotypic constraint. Fitness tradeoffs likely limit the breadth of values for adaptive trait

129 optima, given that extreme trait values will rarely optimize competing functions (33). Notably,

130 the specific mechanisms for constraints on trait values are not specified but are implicit in the

131 application of Ornstein-Uhlenbeck (OU) process to model evolution phenomenologically. 
133 prediction of how the species variation in composite traits $\left(f_{\mathrm{S}}\right.$ and $\left.g_{\max }\right)$ are constrained by their

134 constituent traits $\left(D_{\mathrm{S}}\right.$ and $\left.A_{\mathrm{S}}\right)$, examination of the trait evolution can indicate which hypothesis

135 was supported. The OU model can indicate which composite trait, $f_{\mathrm{S}}$ or $g_{\max }$, is primarily

136 constrained. In both cases, analogous quantitative theory shows that constraint on composite

137 traits imposed by stabilizing selection limits variation in constituent traits(34), and constraint on

$138 f_{\mathrm{S}}$ results in a different covariance structure of $D_{\mathrm{S}}$ versus $A_{\mathrm{S}}$ than a primary constraint on $g_{\max }$.

139 Note that both $f_{\mathrm{S}}$ and $g_{\max }$ show similar mathematical dependence on $D_{\mathrm{S}}$ and $A_{\mathrm{S}}$ :

$$
Z_{S}=\lambda D_{\mathrm{S}} A_{\mathrm{S}}^{\beta}
$$

140 where composite stomatal trait $Z_{\mathrm{S}}\left(\right.$ i.e., $f_{\mathrm{S}}$ or $\left.g_{\max }\right)$ is proportional to the product of constituent

141 stomatal traits, with scaling exponent $\beta$ multiplied by a scalar $\lambda$, which reflects stomatal

142 dimension proportionalities and physical diffusion factors (22). For $g_{\max }, \lambda=b m$ and $\beta=0.5$

143 (Eq. 1); for $f_{\mathrm{S}}, \lambda=1$ and $\beta=1$ (Eq. 2). Since all traits are log-normally distributed ${ }^{31}$, and the

144 OU model assumes Gaussian traits, we log-transformed Eq. 3:

$$
z_{S}=\log (\lambda)+d_{\mathrm{S}}+\beta a_{\mathrm{S}}
$$

145 where lowercase variables indicate log-transformation of uppercase counterparts. Log-

146 transformation also has the advantage of simplifying variance decomposition by linearizing the

147 equation and enables traits measured on different scales to be directly compared in their

148 proportional changes. Using random variable algebra, the variance in $z_{\mathrm{s}}$ is defined as:

$$
\operatorname{Var}\left(z_{S}\right)=\operatorname{Var}\left(\boldsymbol{d}_{\mathrm{S}}\right)+\boldsymbol{\beta}^{2} \operatorname{Var}\left(\boldsymbol{a}_{\mathrm{S}}\right)+2 \boldsymbol{\beta} \operatorname{Cov}\left(\boldsymbol{d}_{\mathrm{S}}, \boldsymbol{a}_{\mathrm{S}}\right)
$$

149 Using the variance-covariance of $d_{\mathrm{S}}$ and $a_{\mathrm{S}}$, we can find the scaling exponent $\beta$ that minimizes $150 \operatorname{Var}\left(z_{S}\right)$ : 


$$
\beta=-\frac{\operatorname{Cov}\left(d_{\mathrm{S}}, a_{\mathrm{S}}\right)}{\operatorname{Var}\left(d_{\mathrm{S}}\right)}
$$

151 Notably, the right-hand side of Eq. 6 is the negative of the ordinary linear regression slope of

152 log-stomatal size against log-density. Thus, for any dataset, $\beta$ can be estimated using ordinary

153 regression methods, but a negative slope estimate will result in a positive value of $\hat{\beta}$. The

154 stomatal-area minimization hypothesis predicts that $\hat{\beta}=1$ because $f_{\mathrm{S}}$ constrains $d_{\mathrm{S}}$ and $a_{\mathrm{S}}(\mathrm{Eq}$.

155 1), whereas the stomatal-area increase hypothesis predicts that $\hat{\beta}=0.5$ because $g_{\max }$ constrains

$156 d_{\mathrm{S}}$ and $a_{\mathrm{S}}$ (Eq. 2). Note that the above prediction assumes that the primary constrained composite

157 trait will also be the least variable composite trait, which allowed to identify the relationship

158 between $\beta$ and trait (co)variance in Eq. 6. We evaluated this assumption using forward-time,

159 individual based, macroevolutionary quantitative genetic simulations (Supplementary

160 Information). In each simulation, 1000 independent lineages evolve toward a moving optimal

161 composite trait until stationarity following an OU process. The simulations confirm that the

162 constrained composite trait is the least variable and that ordinary regression on interspecific trait

163 means can accurately identify the simulated $\beta$. Estimates of $\beta$ are not substantially affected by

164 microevolutionary details about mutational and genetic covariances or geometric constraints on

$165 f_{\mathrm{S}}$ (Fig. S2-S5).

166 We estimated stomatal size-density scaling in 2408 forest plant species from new field-

167 collected samples over 28 sites in China and global synthesis of data from the literature (Fig. 2)

168 and estimated the scaling exponent $\beta$ using OU phylogenetic multiple regression with group

169 (Angiosperm, Pteridophyte, Gymnosperm) and growth form (tree, shrub, herb) as covariates (see

170 Methods).

171 Stomatal size-density scaling among forest plant species was consistent with a primary

172 constraint on $g_{\max }$ (stomatal-area increase hypothesis, $\beta=0.5$ ). Given the variance in stomatal 
173 density, the covariance between size and density among forest species minimizes the variance in

$174 g_{\max }$. This implies that selection for higher $g_{\max }$ results in increased stomatal area allocation, and

175 not minimizing area allocation (Fig. 3). There is no evidence that scaling differs between major

176 groups, Angiosperms, Gymnosperms, and Pteridophytes (Fig. 3a; Table S1), but $g_{\max }$ is 49\% (17-

$17788 \% 95 \% \mathrm{CI} ; P=0.001)$ and $14 \%(1-30 \% 95 \% \mathrm{CI} ; P=0.04)$ higher in Angiosperms than

178 Gymnosperms and Pteridophytes, respectively (Table S2). Trees also have 18\% (8-28\% 95\% CI;

$179 P<0.0001)$ and $48 \%(39-59 \% 95 \% \mathrm{CI} ; P<0.0001)$ greater $g_{\max }$ than shrubs and herbs,

180 respectively (Table $\mathrm{S} 2)$. The across-species mean and variance in $\log \left(g_{\max }\right)$ are nearly invariant

181 across latitude, temperature, and precipitation gradients, indicating that most of the variation in

$182 g_{\max }$ occurs for species of contrasting ecology within rather than between forest sites, a finding

183 similar to that for other key functional traits such as leaf mass per area and wood density (35)

184 (Fig. 4).

185 Our results overturn the prevailing view that the evolution of high $g_{\max }$ across diverse

186 species is constrained by size-density scaling and minimized stomatal area allocation. Instead,

187 the covariance between stomatal size and density supports stomatal area allocation increasing

188 with the evolution of high $g_{\max }$. Thus, limits on the fraction of epidermis allocated to stomatal

$189\left(f_{\mathrm{S}}\right)$ are a secondary consequence of limits on $g_{\max }$. Our novel analysis developed from

190 quantitative genetic and macroevolutionary theory could distinguish the $g_{\max }$ evolution

191 hypotheses. Notably, our $\beta$ exponent for the scaling of $d_{\mathrm{S}}$ and $a_{\mathrm{S}}$ depends on using

192 (phylogenetic) least squares regression, and thus, the results of studies reporting stomatal scaling

193 slopes using standardized major axis (SMA) regression (which minimizes residual variance in

194 both $d_{\mathrm{S}}$ and $a_{\mathrm{S}}$ ) would need to be recalculated to test against our findings (see Supplementary

195 Information). Although estimated scaling using standard phylogenetic regression approaches (see 
196 Methods), it is more appropriate to interpret our results not as minimizing residual variance, but

197 rather estimating the $\beta$ consistent with the covariance structure of stomatal size and density (Fig.

198 1).

199 Our results have at least two important implications for understanding the evolutionary

200 anatomical mechanisms of high $g_{\max }$ and its consequences for the stomatal size-density scaling

201 relationship. First, the finding that size-density scaling does not constrain the evolution of higher

$202 g_{\max }$ implies that stomatal cost is not a constraint on high $g_{\max }$ and thus a different constraint on

203 the evolution of extreme values of $g_{\max }$ across environments. Very high $g_{\max }$ may be rare because

204 the $g_{\text {op }}: g_{\max }$ ratio is constrained in a region of maximal control to respond rapidly to changing

205 environments (36). Additionally or alternatively, a high $g_{\max }$ may also be linked with a high

206 wilting point thereby setting a physical upper limit to leaf gas exchange and a high risk of

207 hydraulic failure (37) if open stomata face transiently high atmospheric drought. Other possible

208 costs include detrimental consequences of high $g_{\max }$ for stomatal movements and diffusion, as

209 well as energetic costs of opening closing more and/or larger stomata $(38,39)$. Future work

210 should prioritize identifying the fitness costs and functional trade-offs that constrain the

211 evolution of high $g_{\max }$. Second, if $g_{\max }$ is the primary constraint, this implies that space allocation

212 to stomata is relatively unimportant, such that plants could allocate a greater fraction of their

213 epidermal area to stomata than they currently do without counterveiling selection. Thus, if

214 stomatal size and density can be manipulated independently, anatomies with the same $g_{\max }$, but

215 different $f_{\mathrm{S}}$, would have similar fitness in the same environment. This finding also clarifies the

216 evolution of stomata across major plant lineages, and refutes the hypothesis that smaller stomata

217 were required to increase $g_{\max }$ in angiosperms (20). All three major land plant lineages have

218 similar variance in $g_{\max }$ (Fig. 3b); angiosperms have higher $g_{\max }$ than gymnosperms and 
219 pteridophytes on average due to their higher $d_{\mathrm{S}}$ for a given $a_{\mathrm{S}}$, not because of differences in the

220 scaling relationship. The higher stomatal density of angiosperms would be linked to increases in

221 leaf water transport capacity, for example, by decreasing the distance between vein and stomata,

222 allowing stomata to stay open ${ }^{40}$. The primary constraint on maximum stomatal conductance

223 appears to be that selection rarely favors extreme values, implying that vegetation and crop

224 models should incorporate nonepidermal costs of extreme trait values to predict optimal $g_{\max }$ for

225 the prediction of photosynthetic carbon gain and transpiratory water loss across scales. 


\section{Methods}

\section{Stomatal trait data from global forests}

228 The stomatal dataset of global forests represents a total of 2408 plant species from natural

229 forests, including novel field data collected from Chinese forest communities and a compilation 230 of published literature values.

231 Our field data were collected from 28 typical forest communities occurring between $18.7^{\circ} \mathrm{N}$ and

$23253.3^{\circ} \mathrm{N}$ latitude in China. The field sites were selected to cover most of the forest types in the

233 northern hemisphere, including cold-temperate coniferous forest, temperate deciduous forest,

234 subtropical evergreen forest, and tropical rain forest (Fig. 2). In total, we sampled 28 forest sites.

235 We used the Worldclim database (40) to extract additional data on mean annual temperature

236 (MAT) and precipitation (MAP) over the period 1960-1990 using latitude and longitude. Among

237 these forests, mean annual temperature (MAT) ranged from $-5.5-23.2{ }^{\circ} \mathrm{C}$, and mean annual

238 precipitation (MAP) varied from 320 to $2266 \mathrm{~mm}$. The field investigation was conducted in July-

239 August, during the peak period of growth for forests. Sampling plots were located within well-

240 protected national nature reserves or long-term monitoring plots of field ecological stations, with

241 relatively continuous vegetation. Four experimental plots $(30 \times 40 \mathrm{~m})$ were established in each

242 forest.

Leaves from trees, shrubs, and herbs were collected within and around each plot. For trees,

244 mature leaves were collected from the top of the canopy in four healthy trees and mixed as a

245 composite sample. Eight to 10 leaves from the pooled samples were cut into roughly $1.0 \times 0.5$

$246 \mathrm{~cm}$ pieces along the main vein, and were fixed in formalin-aceto-alcohol (FAA) solution (5 ml

$24738 \%$ formalin, $90 \mathrm{ml} 75 \%$ ethanol, $5 \mathrm{ml} 100 \%$ glacial acetic acid, and $5 \mathrm{ml} 37 \%$ methanol) 
248 (41). In the laboratory, three small pieces were randomly sampled, and each replicate was

249 photographed twice using a scanning electron microscopy (Hitachi SN-3400, Hitachi, Tokyo,

250 Japan) on the lower surface at different positions. We focused on the lower epidermis (42),

251 because a previous study has demonstrated that most of leaf upper epidermis has no stomata for

252 forest plants (43).

253 In each photograph, the number of stomata was recorded, and $D_{\mathrm{S}}$ was calculated as the

254 number of stomata per unit leaf area. Simultaneously, five typical stomata were selected to

255 measure stomatal size using an electronic image analysis equipment (MIPS software, Optical

256 Instrument Co. Ltd., Chongqing, China).

257 Peer-reviewed papers on leaf stomata were collected using an all-databases search of Web

258 of Science (www.webofknowledge.com) from 1900 to 2018 using "forest" and "stomata" as a

259 topic, in line with the principle of "natural forest, non-intervention, species name" (i.e. we did

260 not use data from controlled experiments or where taxonomic data was missing). A total of 90

261 papers (see Supporting Table S3) which met our requirements, yielding $\mathrm{D}_{\mathrm{S}}$ and $L$ measurements

262 from 413 plant species (Fig. 2) from which we calculated $g_{\max }$ and $f_{\mathrm{S}} . f_{\mathrm{S}}$ is proportional to the

263 stomatal pore area index (SPI), which defined as the product of $D_{\mathrm{S}}$ and stomatal length $(L)$

264 squared (25), because $A_{\mathrm{S}}=m L^{2}(22)$.

265 We calculated $g_{\max }\left(\right.$ Equation 1) to water vapor at a reference leaf temperature $\left(\mathrm{T}_{\text {leaf }}=25^{\circ}\right.$

266 C) following Sack and Buckley (22). They defined a biophysical and morphological constant as:

$$
\begin{gathered}
b=\frac{D_{\mathrm{wv}}}{v} \\
m=\frac{\pi c^{2}}{j^{0.5}(4 h j+\pi c)}
\end{gathered}
$$


$267 b$ is the diffusion coefficient of water vapor in air $\left(D_{\mathrm{wv}}\right)$ divided by the kinematic viscosity of dry

268 air $(v) . D_{\mathrm{wv}}=2.49 \times 10^{-5} \mathrm{~m}^{2} \mathrm{~s}^{-1}$ and $v=2.24 \times 10^{-2} \mathrm{~m}^{3} \mathrm{~mol}^{-1}$ at $25^{\circ}(44)$. For kidney-

269 shaped guard cells, $c=h=j=0.5$; for dumbbell-shaped guard cells in the Poaceace, $c=h=$

$270 \quad 0.5$ and $j=0.125$. We used the species average $g_{\max }$ and $f_{\mathrm{S}}$ for all analyses.

271 Phylogenetic regression

272 By positing that the least variable composite of stomatal size and density indicates the trait

273 with the most constraint (Fig. 1), we identify a new way to estimate the scaling exponent $\beta$ (Eq.

274 6) using linear regression estimates, and also accounted for phylogenetic nonindependence. We

275 used the Plant List (http://www.theplantlist.org) to confirm species names, then we assembled a

276 synthetic phylogeny using S.PhyloMaker (45). We fitted phylogenetic regression models using

277 the phylolm version 2.6 package in $\mathrm{R}(46)$. As we derived in the main text, the scaling exponent

$278 \beta$ can be estimated from the slope of the regression of $a_{\mathrm{S}}$ on $d_{\mathrm{S}}$, where $\hat{\beta}=-$ slope. We estimated

279 separate scaling exponents for major groups, Angiosperms, Pteridophytes, and Gymnosperms.

280 We also estimated different intercepts, corresponding with different average $g_{\max }$ values, for

281 functional types (herbs, shrubs, and trees) and grasses, because of their unique stomatal anatomy.

282 We used the "OUrandomRoot" model of trait evolution. 95\% confidence intervals for all

283 parameters were estimated from 1000 parametric bootstrap samples generated by simulating

284 from the best-fit model and re-fitting. $P$-values for coefficients are based on $t$-tests. We used the

285 same methods to test whether $g_{\max }$ (log-transformed for homoskedasticity) was affected by

286 |latitude|, MAP, MAT, group (Angiosperms, Pteridophytes, Gymnosperms), and/or functional

287 type (herb, shrub, tree). One gymnosperm species, Torreya fargesii, had substantially lower

288 stomatal size than would be predicted from its density (Fig. 3a). There results of the paper did

289 not change if this outlier was excluded because the confidence intervals for stomatal-density 
bioRxiv preprint doi: https://doi.org/10.1101/2021.04.25.441252; this version posted September 18, 2021. The copyright holder for this preprint (which was not certified by peer review) is the author/funder. All rights reserved. No reuse allowed without permission.

290 scaling are very wide for Gymnosperms regardless. Therefore, we excluded this species from

291 statistical analyses but show it in the figure for completeness. All data were analyzed in R

292 (47)version 4.0.5

293

294

295

296 


\section{References}

299 1. J. A. Berry, D. J. Beerling, P. J. Franks, Stomata: key players in the earth system, past and present. Current Opinion in Plant Biology 13, 232-239 (2010).

2. W. Cramer et al., Global response of terrestrial ecosystem structure and function to $\mathrm{CO}_{2}$ and climate change: results from six dynamic global vegetation models. Global Change Biology 7, 357-373 (2001).

3. M. Haworth, C. Elliott-Kingston, J. C. McElwain, Stomatal control as a driver of plant evolution. Journal of Experimental Botany 62, 2419-2423 (2011).

4. G. J. Jordan, R. J. Carpenter, A. Koutoulis, A. Price, T. J. Brodribb, Environmental adaptation in stomatal size independent of the effects of genome size. New Phytologist 205, 608-617 (2015).

5. A. M. Hetherington, F. I. Woodward, The role of stomata in sensing and driving environmental change. Nature 424, 901-908 (2003).

6. P. J. Franks et al., Sensitivity of plants to changing atmospheric $\mathrm{CO}_{2}$ concentration: from the geological past to the next century. New Phytologist 197, 1077-1094 (2013).

7. M. Haworth, C. Elliott-Kingston, J. C. McElwain, Co-ordination of physiological and morphological responses of stomata to elevated $\mathrm{CO}_{2}$ in vascular plants. Oecologia 171, 71-82 (2013).

8. J. C. Ordoñez et al., A global study of relationships between leaf traits, climate and soil measures of nutrient fertility. Global Ecology and Biogeography 18, 137-149 (2009).

9. Z. Y. Yuan, H. Y. H. Chen, Global-scale patterns of nutrient resorption associated with latitude, temperature and precipitation. Global Ecology and Biogeography 18, 11-18 (2009).

10. S. Díaz et al., The global spectrum of plant form and function. Nature 529, 167-171 (2016).

11. G. T. Freschet et al., Climate, soil and plant functional types as drivers of global fine-root trait variation. Journal of Ecology 105, 1182-1196 (2017).

12. H. T. Brown, F. Escombe, Static diffusion of gases and liquids in relation to the assimilation of carbon and translocation in plants. Proceedings of the Royal Society of London 67, 124-128 (1901).

13. J.-Y. Parlange, P. E. Waggoner, Stomatal dimensions and resistance to diffusion. Plant Physiology 46, 337-342 (1970).

14. P. J. Franks, G. D. Farquhar, The effect of exogenous abscisic acid on stomatal development, stomatal mechanics, and leaf gas exchange in Tradescantia virginiana. Plant Physiology 125, 935-942 (2001).

15. A. Vatén, D. C. Bergmann, Mechanisms of stomatal development: an evolutionary view. EvoDevo 3, 11 (2012).

16. J. C. McElwain, C. Yiotis, T. Lawson, Using modern plant trait relationships between observed and theoretical maximum stomatal conductance and vein density to examine patterns of plant macroevolution. New Phytologist 209, 94-103 (2016).

17. M. À. Conesa, C. D. Muir, A. Molins, J. Galmés, Stomatal anatomy coordinates leaf size with Rubisco kinetics in the Balearic Limonium. AoB PLANTS 12 (2019). 
18. M. Murray et al., Consistent relationship between field-measured stomatal conductance and theoretical maximum stomatal conductance in c3 woody angiosperms in four major biomes. International Journal of Plant Sciences 181, 142-154 (2020).

19. A. G. Weiss, Untersuchungen über die Zahlen-und Grössenverhältnisse der Spaltöffnungen (Jahrbu“cher für Wissenschaftliche Botanik, 1865), vol. 4.

20. P. J. Franks, D. J. Beerling, Maximum leaf conductance driven by $\mathrm{CO}_{2}$ effects on stomatal size and density over geologic time. Proceedings of the National Academy of Sciences 106, 10343-10347 (2009).

21. G. J. Dow, J. A. Berry, D. C. Bergmann, The physiological importance of developmental mechanisms that enforce proper stomatal spacing in Arabidopsis thaliana. New Phytologist 201, 1205-1217 (2014).

22. L. Sack, T. N. Buckley, The developmental basis of stomatal density and flux. Plant Physiology 171, 2358-2363 (2016).

23. E. J. Salisbury, On the causes and ecological significance of stomatal frequency, with special reference to the woodland flora. Philosophical Transactions of the Royal Society of London Series B 216, 1-65 (1928).

24. R. M. Deans, T. J. Brodribb, F. A. Busch, G. D. Farquhar, Optimization can provide the fundamental link between leaf photosynthesis, gas exchange and water relations. Nature Plants 6, 1116-1125 (2020).

25. L. Sack, P. D. Cowan, N. Jaikumar, N. M. Holbrook, The 'hydrology' of leaves: coordination of structure and function in temperate woody species. Plant, Cell \& Environment 26, 1343-1356 (2003).

26. S. F. Bucher et al., Inter- and intraspecific variation in stomatal pore area index along elevational gradients and its relation to leaf functional traits. Plant Ecology 217, 229-240 (2016).

27. Stephanie Dunbar-Co, Margaret J. Sporck, Lawren Sack, Leaf trait diversification and design in seven rare taxa of the hawaiian plantago radiation. International Journal of Plant Sciences 170, 61-75 (2009).

28. M. W. Pennell, L. J. Harmon, An integrative view of phylogenetic comparative methods: connections to population genetics, community ecology, and paleobiology. Annals of the New York Academy of Sciences 1289, 90-105 (2013).

29. T. F. Hansen, Stabilizing selection and the comparative analysis of adaptation. Evolution 51, 1341-1351 (1997).

30. J. C. Uyeda, L. J. Harmon, A novel bayesian method for inferring and interpreting the dynamics of adaptive landscapes from phylogenetic comparative data. Systematic Biology 63, 902-918 (2014).

31. F. C. Boucher, V. Démery, E. Conti, L. J. Harmon, J. Uyeda, A general model for estimating macroevolutionary landscapes. Systematic Biology 67, 304-319 (2017).

32. R. Lande, Natural selection and random genetic drift in phenotypic evolution. Evolution 30, 314-334 (1976).

33. L. Sack, T. N. Buckley, Trait multi-functionality in plant stress response. Integrative and Comparative Biology 60, 98-112 (2019).

34. B. Walsh, M. W. Blows, Abundant Genetic Variation + Strong Selection $=$ Multivariate Genetic Constraints: A Geometric View of Adaptation. Annual Review of Ecology, Evolution, and Systematics 40, 41-59 (2009). 
35. Mark Westoby, Daniel S. Falster, Angela T. Moles, a. Peter A. Vesk, I. J. Wright, Plant ecological strategies: Some leading dimensions of variation between species. Annual Review of Ecology and Systematics 33, 125-159 (2002).

36. P. J. Franks, I. J. Leitch, E. M. Ruszala, A. M. Hetherington, D. J. Beerling, Physiological framework for adaptation of stomata to $\mathrm{CO}_{2}$ from glacial to future concentrations. Philosophical Transactions of the Royal Society B: Biological Sciences 367, 537-546 (2012).

37. C. Henry et al., A stomatal safety-efficiency trade-off constrains responses to leaf dehydration. Nature Communications 10, 3398 (2019).

38. H. J. de Boer et al., Optimal allocation of leaf epidermal area for gas exchange. New Phytologist 210, 1219-1228 (2016).

39. G. D. Farquhar, I. R. Cowan, E. Zeiger, Stomatal Function. G. D. Farquhar, I. R. Cowan, E. Zeiger, Eds. (Stanford University Press, Stanford, 1987), pp. 520.

40. R. J. Hijmans, S. E. Cameron, J. L. Parra, P. G. Jones, A. Jarvis, Very high resolution interpolated climate surfaces for global land areas. International Journal of Climatology 25, 1965-1978 (2005).

41. N. He et al., Variation in leaf anatomical traits from tropical to cold-temperate forests and linkage to ecosystem functions. Functional Ecology 32, 10-19 (2018).

42. C. Liu et al., Variation of stomatal traits from cold temperate to tropical forests and association with water use efficiency. Functional Ecology 32, 20-28 (2018).

43. C. D. Muir, Light and growth form interact to shape stomatal ratio among British angiosperms. New Phytologist 218, 242-252 (2018).

44. J. L. Monteith, M. H. Unsworth, "Chapter 2 - Properties of Gases and Liquids" in Principles of Environmental Physics (Fourth Edition), J. L. Monteith, M. H. Unsworth, Eds. (Academic Press, Boston, 2013), https://doi.org/10.1016/B978-0-12-3869104.00002-0, pp. 5-23.

45. H. Qian, Y. Jin, An updated megaphylogeny of plants, a tool for generating plant phylogenies and an analysis of phylogenetic community structure. Journal of Plant Ecology 9, 233-239 (2015).

46. L. Ho, C. Ané, A linear-time algorithm for Gaussian and non-Gaussian trait evolution models. Syst Biol 63, 397-408 (2014).

47. R. C. Team (2021) R: A language and environment for statistical computing. Foundation for statistical computing. . in $R$ : A language and environment for statistical computing. Foundation for statistical computing. (Vienna, Austria, Vienna, Austria). 


\section{Acknowledgements}

424 Financial support was supported by the National Natural Science Foundation of China

425 (31988102, 31770655, 31870437), the National Key R\&D Program of China

426 (2017YFA0604803), the second Tibetan Plateau Scientific Expedition and Research Program

427 (2019QZK060202), The Chinese Academy of Sciences Strategic Priority Research Program

428 (XDA23080401), US National Science Foundation 1929167 (to CDM), and the Project funded

429 by China Postdoctoral Science Foundation (2020M680663). We thank "Functional Trait

430 Database of Terrestrial Ecosystems in China (China_Trait)" for sharing data, further information

431 for other materials should contact to N.P. He (henp@igsnrr.ac.cn). There are no conflicts of

432 interest to declare.

\section{Author contributions}

436 N.H. and G.Y. designed field sampling; N.H., C.D.M, and C.L. conceived the initial ideas; C.L.,

437 N.H., Y.L. J.Z., Z.Z., M.L. and L.X collected the data; C.L. wrote the first draft, and C.D.M.

438 contributed the final mathematical derivations, data analysis, and wrote the final manuscript; L.S.,

439 H.J.B., C.L., N.H., G.Y., and X.H. revised the manuscript. All authors gave final approval for 440 publication. 
bioRxiv preprint doi: https://doi.org/10.1101/2021.04.25.441252; this version posted September 18, 2021. The copyright holder for this preprint (which was not certified by peer review) is the author/funder. All rights reserved. No reuse allowed without permission.

\section{Supplementary information}

\section{$443 \quad$ Figures}



density are geometrically possible

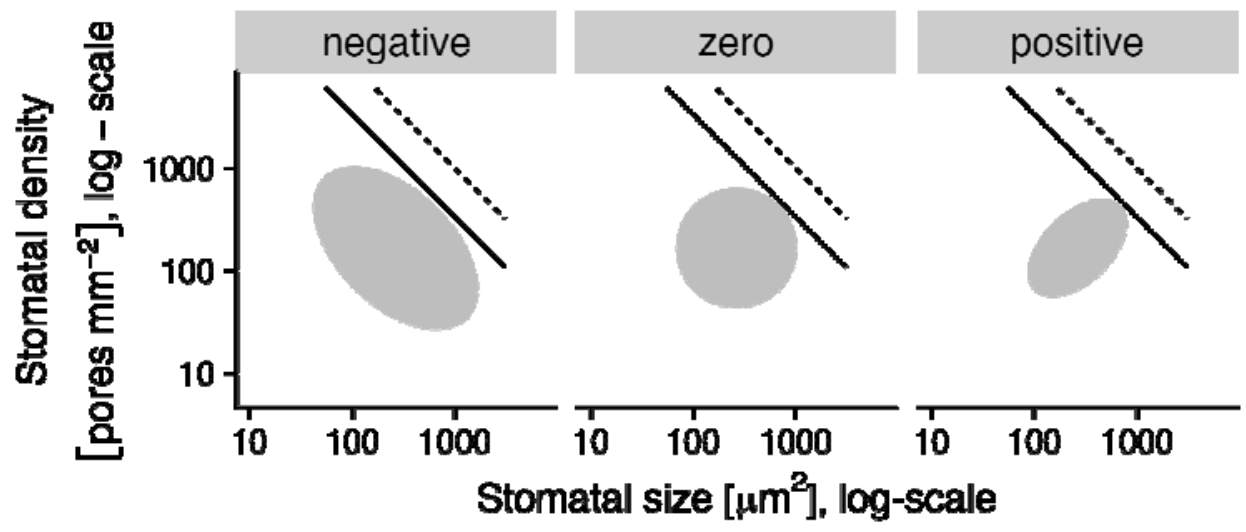

b Hypothesis: covariation in stomatal size and density is shaped by constraint on a composite trait

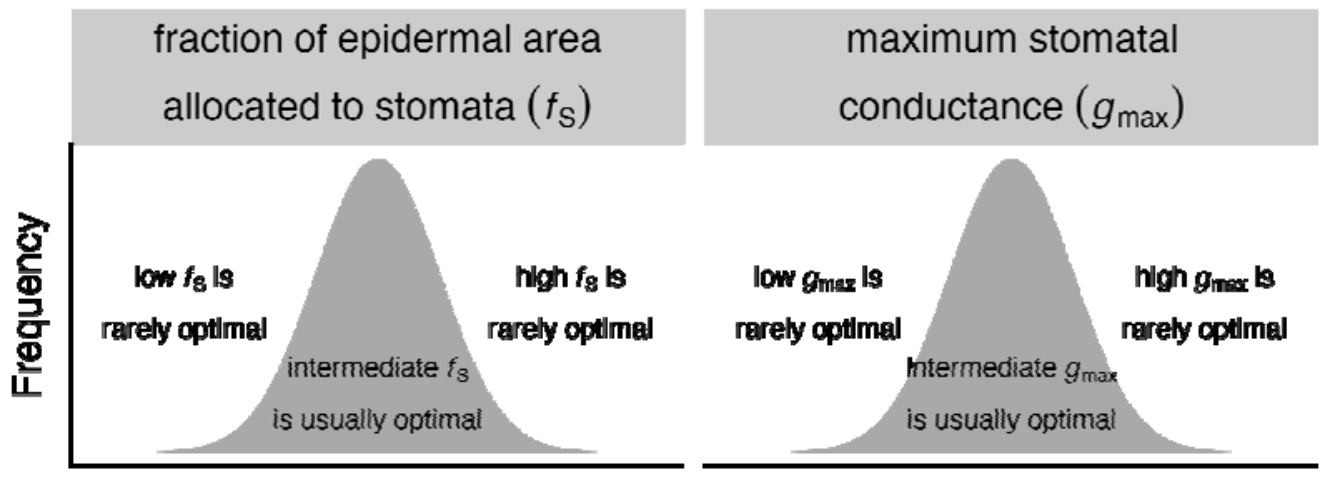

Optimal composite trait value

c Prediction: stomatal size-density covariation depends on which composite trait is constrained

stomatal - area minimization

$f_{\mathrm{S}}$ constrained, $\beta=1.0$

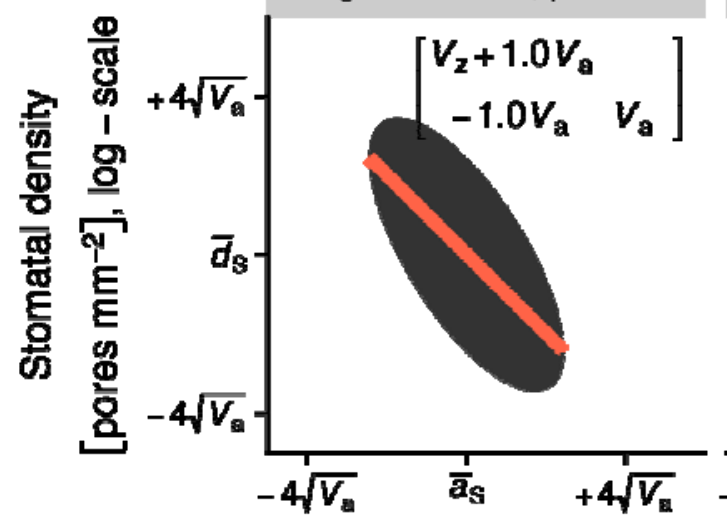

stomatal - area increase

$g_{\text {max }}$ constrained, $\beta=0.5$

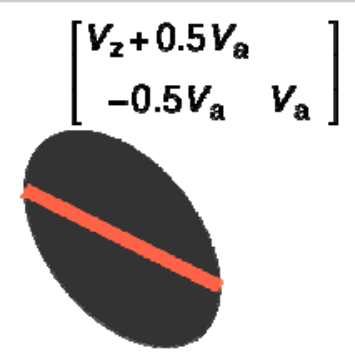

Stomatal size $\left[\mu \mathrm{m}^{2}\right]$, log-scale 


\section{Fig. 1 | Competing hypotheses for stomatal size-density scaling make different predictions}

446 about the trait covariance structure. Maximum stomatal conductance $\left(g_{\max }\right)$ and the fraction of

447 epidermal area allocated to stomata $\left(f_{\mathrm{S}}\right)$ are composite traits determined by stomatal density and

448 size. On a log-scale, they are the sum of log-stomatal density $\left(d_{\mathrm{S}}\right)$ and log-stomatal size $\left(a_{\mathrm{S}}\right)$

449 times a scaling exponent $(\beta), 0.5$ for $g_{\max }$ and 1.0 for $f_{\mathrm{S}}$ (see Methods). a. Many scaling

450 relationships between stomatal size and density are possible as long as $f_{\mathrm{S}}$ does not exceed 1

451 (dashed line) or more realistically a value less than 1 to allow space between stomata (solid line,

$452 f_{\mathrm{S}}=0.34$, the maximum value in our data set). The grey ellipses represent different possible

453 scaling relationships with the same mean trait values in our data set $\left(\bar{A}_{\mathrm{S}}=263 \mu \mathrm{m}^{2}, \bar{D}_{\mathrm{S}}=\right.$

454168 pores $\mathrm{mm}^{-2}$ ). These are $95 \%$ quantile of covariance ellipses for a bivariate normal with

455 trait correlations of $-0.5,0$, and 0.5 and trait variances of $0.75,0.55$, and 0.45 for 'negative',

456 'zero', and 'positive' relationships, respectively. b. We hypothesized that size-density scaling is

457 determined by constraint on either $g_{\max }$ (stomatal-area increase; left panel) or $f_{\mathrm{S}}$ (stomatal-area

458 minimization; right panel). Under either hypothesis, the optimal composite trait varies but

459 extreme values of the composite trait are rarely optimal. c. Both hypotheses predict negative

460 size-density scaling but with different covariance relationships. If the interspecific means $\left(\bar{d}_{\mathrm{S}}, \bar{a}_{\mathrm{S}}\right)$

461 and variances $\left(V_{d}, V_{a}\right)$ of stomatal density and size, respectively, are measured, the covariance

462 between them $\left(V_{d, a}\right)$ is equal to $-\beta V_{a}$. Under the stomatal-area increase (left panel) and stomatal-

463 area minimization (right panel) hypotheses, $\beta$ should be 0.5 and 1 , respectively. The ellipse is the

4640.95 quantile of covariance ellipse associated with the covariance matrix (upper right corner of

465 the plot); the orange line is the scaling exponent fit through the constituent trait means. 


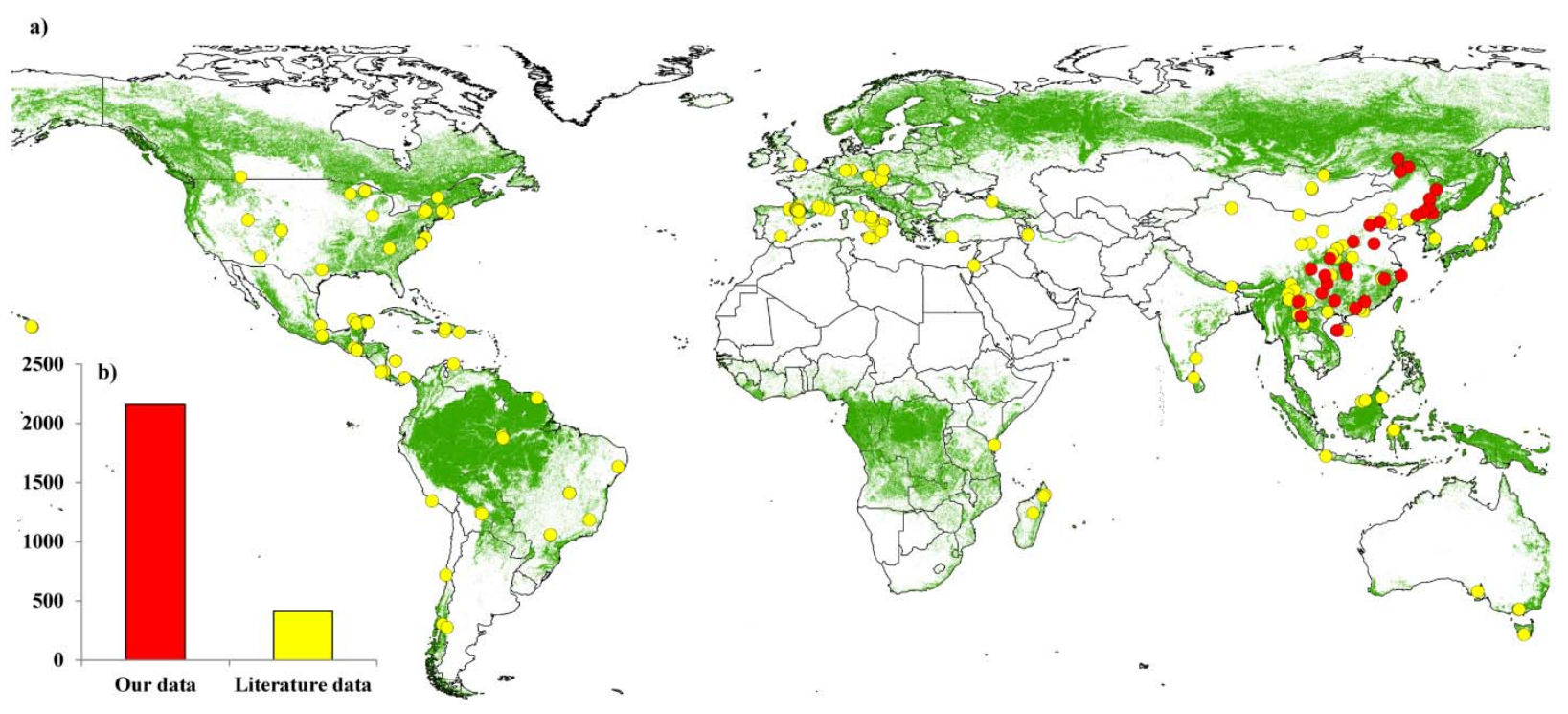


a

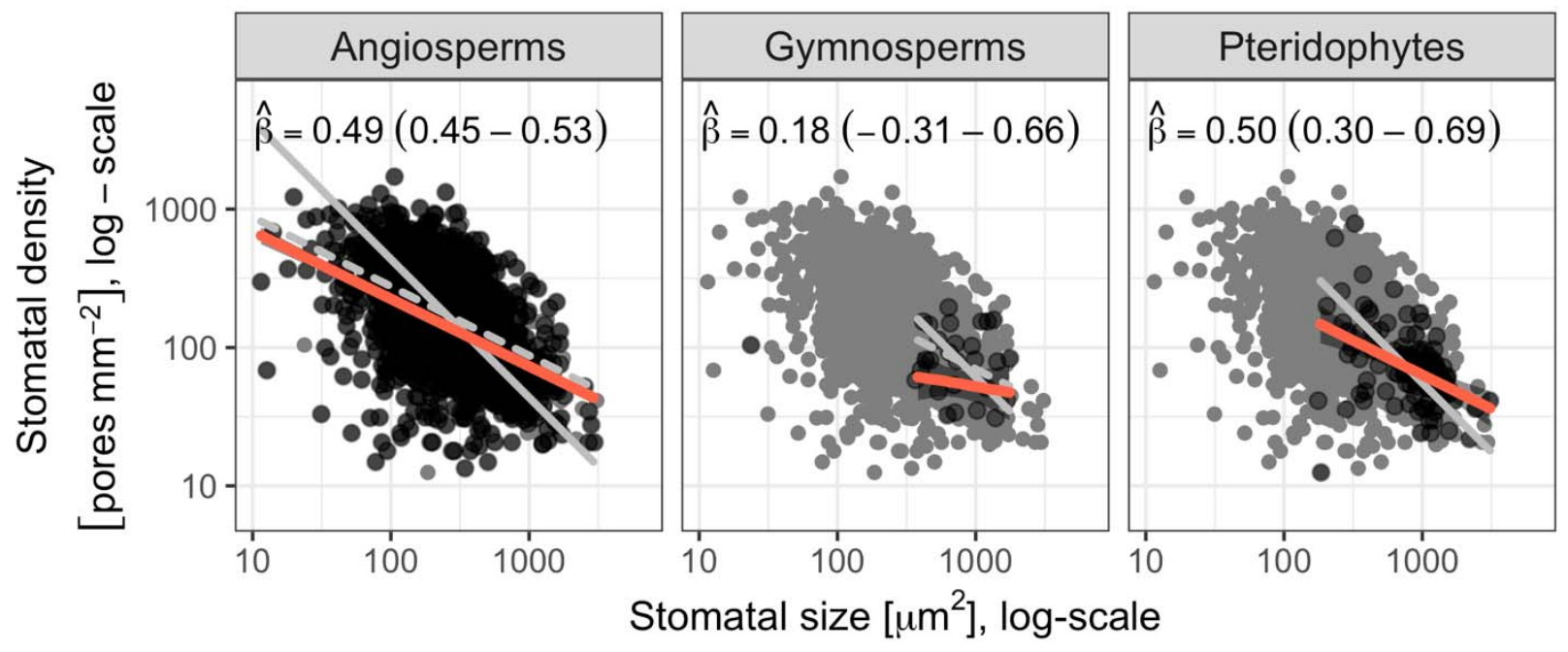

b

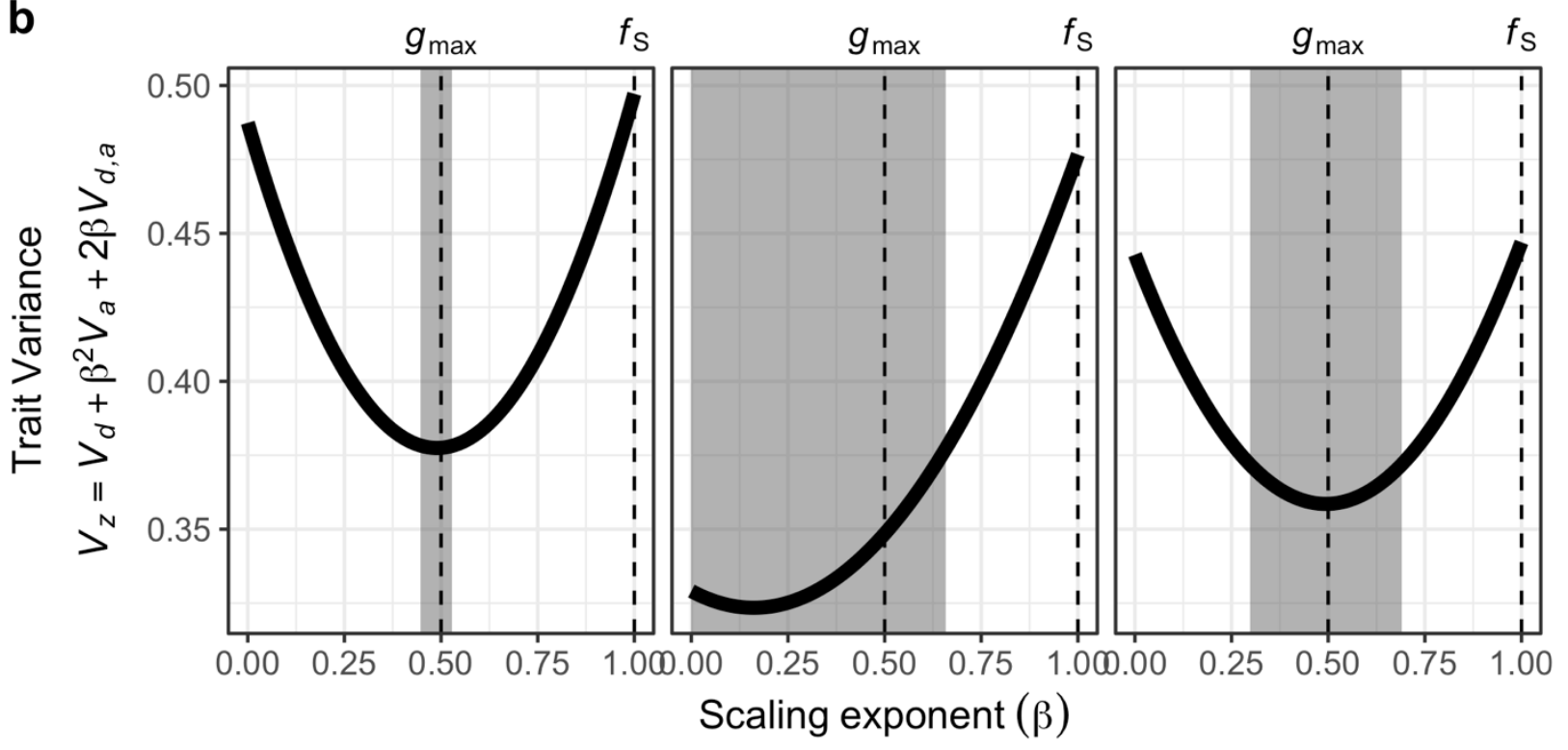

471 Fig. 3 | Stomatal size-density scaling is consistent with stomata-area increase but not area-

472 minimization. a. In both angiosperms (left panel) and pteridophytes (right panel), the scaling

473 exponent ( ) estimated as the phylogenetic linear regression slope of stomatal size against

474 density (Methods) is close to 0.5 as predicted by the stomatal-area increase hypothesis, but much

475 less than 1.0, as predicted by the stomatal-area minimization hypothesis. For comparison, thin

476 gray lines in the background show predicted slopes for each group when $=1.0$ (solid line) and 
$477 \beta=0.5$ (dashed line). The bootstrap 95\% confidence intervals are in parentheses and shown

478 graphically by the width of the grey rectangle in $\mathbf{b}$. Dark points represent species mean trait

479 values from the focal group; grey background points are from all groups for comparison. Orange

480 line and ribbon are the estimated phylogenetic regression line and the $95 \%$ bootstrap confidence

481 intervals. Scaling in gymnosperms (middle panel) is not significantly different from 0 or 0.5 , but

482 the confidence intervals do not include 1.0. b. The variance of the composite trait $\left(V_{z}\right)$ is

483 minimized near $\beta=0.5$, as predicted under the stomatal-area increase hypothesis (dashed-line

484 under $g_{\max }$ ) but not where $\beta=1.0$ as predicted by the stomatal-area minimization hypothesis

485 (dashed-line under $f_{\mathrm{S}}$ ). 


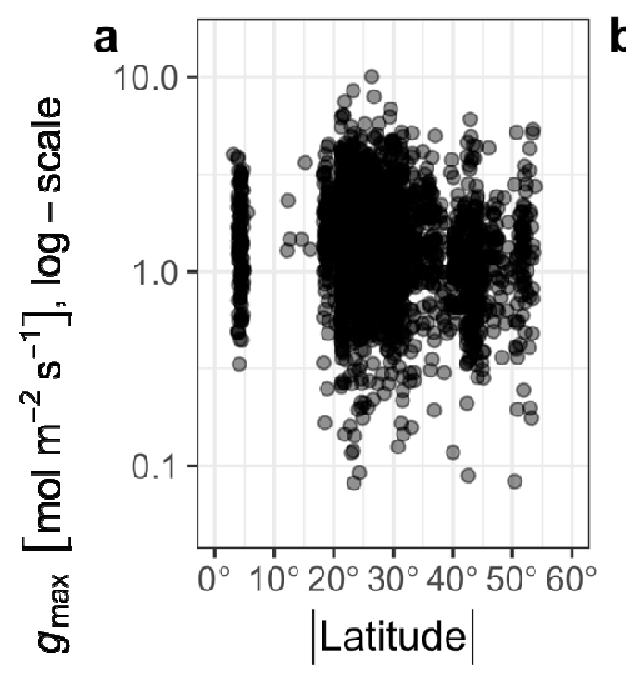

488

489

490

491

492

493

494

495 variables is only 0.11.

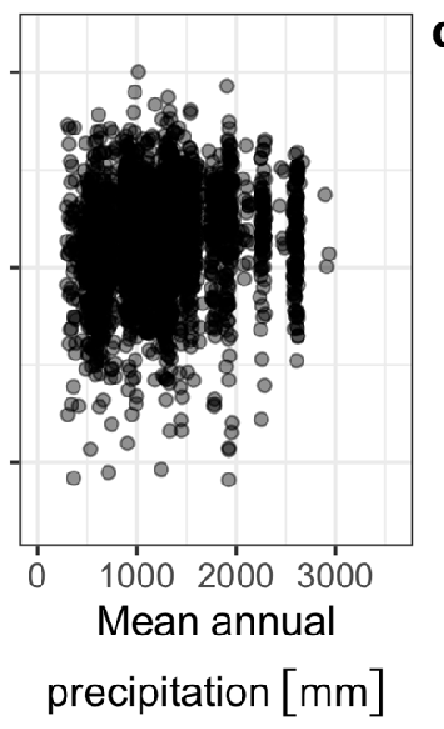

C

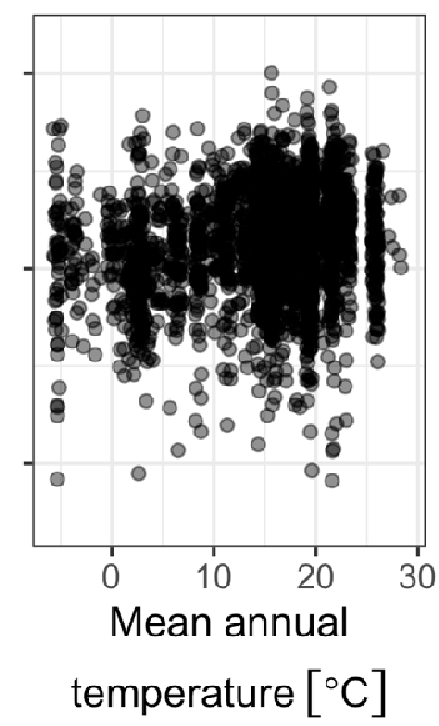

Fig. 4 | Anatomical maximum stomatal conductance varies little with latitude, mean annual precipitation, or mean annual temperature. Each point is the species' mean |latitude|(a.), mean annual precipitation (b.), or mean annual temperature (c.) on the $x$-axis and the maximum stomatal conductance $\left(g_{\max }\right)$ on the $y$-axis (log-scale). Based on phylogenetic multiple regression, the relationship between $\log \left(g_{\max }\right)$ and mean |latitude| $(P=0.69)$ and mean annual temperature $(P$ $=0.10)$ are not significant; the relationship with mean annual precipitation is significant $(P=$ 0.009 ) but weak since the total model $R^{2}$ including all climate, lineage, and growth explanatory 